\title{
EFFECT OF SOME FOLIAR APPLICATION NUTRIENT SPRAYS ON THE PRODUCTIVITY AND FRUIT QUALITY OF KALAMATA OLIVE CULTIVAR
}

(Received : 23.7. 2014)

By

\author{
Sh. M. M. El-Sharkawy, I. M. S. Osman and E. G. Mikhail
}

Horticulture Research Institute, Agricultural Research Center, Giza, Egypt

\begin{abstract}
This investigation was carried out during 2012 and 2013 seasons on 6 year old olive trees cv. Kalamata. Selected trees were planted at $6 \times 4$ meters apart in a sandy soil; subjected to drip irrigation; uniform in shape and size as much as possible and received regularly the recommended horticultural practices according to the recommendations of the Horticulture Research Institute. Kalamata olive trees were foliar sprayed with calcium chloride at 1 or $2 \%$; potassium chloride at 2 or $4 \%$, and urea at $2 \%$ each alone or in combination at full bloom. Obtained data indicated that all tested treatments increased fruit set, the number of fruits per tree, yield (kilogram per tree) and improved fruit quality (fruit weight, fruit dimensions flesh/pit ratio and oil content) while, decreased both June and pre-harvest drop in comparison with the control. In addition to improve NPK status of Kalamata olive leaves.

The most effective treatments on tree fruiting were the combinations of 2 and $4 \%$ potassium chloride with $2 \%$ urea. Regarding, fruit quality, the two concentrations of calcium chloride or potassium chloride alone or supplemented with $2 \%$ urea, improved fruit quality. Besides, 2 or $4 \%$ potassium chloride induced the highest values of fruit oil percentage as fruit fresh weight, while as dry weight, the treatment of $1 \%$ calcium chloride associated with $2 \%$ urea induced the highest values. Regarding leaf NPK content, the treatment of $2 \%$ calcium chloride increased leaf nitrogen content in the first season, while, treatment of $2 \%$ potassium chloride $+2 \%$ urea was the most effective treatment in the second season. Either $2 \%$ or $4 \%$ potassium chloride treatments increased leaf P content during 2012 $\& 2013$ seasons, whereas leaf $\mathrm{K}$ content was not affected with the tested treatments in the first season, while in second season the treatment of $1 \%$ calcium chloride was the most effective treatment. This study recommended that spraying olive trees cv. Kalamata with $1 \%$ or $2 \%$ calcium chloride; 2 or $4 \%$ potassium chloride and $2 \%$ urea, each alone or in combination, at full bloom increased tree fruiting; improved fruit quality and increased fruit oil content.
\end{abstract}

Keywords: calcium chloride, fruit quality, fruit set, leaf NPK, oil content, olive, potassium chloride, urea, yield.

\section{INTRODUCTION}

In Egypt, table olive cultivars play an important role in economics of the growers and countries where most of the olive production is consumed as pickling products. Thereby, poor fruit set, high fruit shedding and consequently poor yields are considered critical factors for growing table cultivars.

Kalamata is a pickling olive cultivar, where high fruit set and consequently the number of fruits per tree with good quality is essential factor to gain high income. Many researches mentioned that Calcium, Nitrogen and Potassium play important roles, in fruit set and fruit retention. Foliar-applied calcium, alone or in combination, has been shown to enhance acclimation or repair of stress-injured tissues (Andrews, 2002). Moreover, Porro et al. (2002) confirmed that foliar nutritional sprays can be a useful management practice to enhance fruit nutrient status and consequent fruit quality.

Olive leaves and stems represent storage organs for nitrogen and release it in response to the metabolic demands of developing reproductive and vegetative organs (FernandezEscobar et al., 2004). Besides, Freeman et al., 2005 reported that under nitrogen deficiency, fruit set, yield and shoot growth are negatively 
influenced. In addition, Therios, 2006 mentioned that nitrogen was found to increase the proportion of hermaphrodite flowers, and low nitrogen concentration in the leaves led to the formation of staminate flowers and therefore decreased the potential level of fruit set in olive trees.

Potassium plays an important role in improving fruit quality of olive trees. In this regards, Ben Mimoum et al. (2004) reported that foliar treatments of potassium on olive trees increased fruit weight, pit ratio and polyphenol. Also, Hussein (2008) mentioned that potassium fertigation improved fruit and fruit oil content of Mazanillo olive cultivar.

Furthermore, many authors reported the positive effect of calcium on fruit quality of olive. Desouky et al. (2009) found that spraying olive trees with $2 \%$ calcium chloride at full bloom and 15 days later increased fruit oil content and improved oil quality. In addition, Haj Sulaiman (2009) reported that spraying olive trees with calcium carbonate increased fruit weight and size; percentage and thickness of flesh and the oil content. In addition, recent data indicate the importance of chloride $\left(\mathrm{Cl}^{-}\right)$transfer across the plasma membrane for pollen germination and pollen tube growth (Tavares et al., 2011).

The aim of this investigation was to study the effect of calcium chloride, potassium chloride and urea foliar fertilization each alone or in different combinations, on fruiting, fruit physical characteristics, fruit oil content and on leaf NPK content.

\section{MATERIAL AND METHODS \\ 2.1. Plant material and treatments}

The present study was carried out during 2012 and 2013 seasons on kalamata olive trees (6 year old), planted in a private farm at Kilometer 64 from Cairo - Alexandria desert road. Selected trees were uniform in shape and size as much as possible and planted at $6 \times 4$ meters apart in a sandy soil; subjected to drip irrigation and received regularly the recommended horticultural practices according to recommendation of the Horticulture Research Institute (El-Sayed and Abou Shanab, 2011).

The selected trees were arranged in a complete randomized design with three replicates per treatment (one tree /replicate). Foliar sprays were applied at full bloom, the study was conducted according to the following treatments:

1- Control (untreated trees).
2- $1 \%$ Calcium chloride $21 \%$.

3- $2 \%$ Calcium chloride $21 \%$.

4- $2 \%$ Urea.

5- $2 \%$ Potassium chloride.

6- $4 \%$ Potassium chloride.

7- $1 \%$ Calcium chloride $21 \%+2 \%$ urea.

8- $2 \%$ Calcium chloride $21 \%+2 \%$ urea.

9- $2 \%$ Potassium chloride $+2 \%$ urea.

10- $4 \%$ Potassium chloride $+2 \%$ urea.

\subsection{Data analysis}

The obtained data were handled as follows

\subsubsection{Tree Fruiting}

Twenty shoots were randomly labeled and numbers of their flowers were counted. Number of fruit set at the beginning of May, the number of fruits in mid June and before harvesting at mid October, were counted and fruit set percentage and both June and pre-harvest drop percentages were calculated. In addition, at harvest time, the number of fruits per tree (fruits retention) were counted and the yield (as kilogram per tree) was calculated.

- The following equations were used for calculations:

Fruit set $(\%)=\frac{\text { Number of set fruitlets }}{\text { Total No of flowers at full bloom }} \times 100$

June drop $(\%)=\frac{\text { Number of dropped fruits until mid Iune }}{\text { Number of set fruitlets }} \times 100$

Pre harvest drop $(\%)=\frac{\text { Number of dropped fruits before harvest }}{\text { Number of fruits after [une drop }} \times 100$

\subsubsection{Fruit physical characteristics}

Thirty fruits per each tree were randomly picked for carrying out the fruit quality measurements:

Fruit length $(\mathrm{cm})$, fruit diameter $(\mathrm{cm})$, fruit shape index, fruit weight $(\mathrm{g})$, flesh weight $(\mathrm{g})$, seed weight $(\mathrm{g})$ and consequently, flesh/pit ratio were calculated

\subsubsection{Fruit oil percentage}

It was determined by extracting the oil from the fruits either as fresh or dry weight by soxhalt extraction apparatus using petroleum ether at 60$80^{\circ} \mathrm{C}$ boiling point as described by A.O.A.C. (1980).

\subsubsection{Leaf NPK determination}

Leaf nitrogen content was determined using micro-Kjeldahle method as described by A.O.A.C. (1980). Leaf P content was determined according to Wide et al. (1985). Leaf K content 
was determined by Atomic Absorption Spectrophotometer (Chapman and Pratt, 1978).

\section{Statistical analysis}

The obtained data were subjected to analysis of variance (ANOVA) utilizing a randomized complete block design according to Snedecor and Cochran (1980). Differences between treatments were compared by Duncan's multiple range tests (1955).

\section{RESULTS AND DISCUSSION \\ 3.1. Tree fruiting}

Data in Table (1) show, the effect of calcium chloride; potassium chloride and urea, alone or in combination, on fruit set, June drop; pre harvest drop, the number of fruits per tree and yield as kg. per tree of Kalamata olive cultivar. during 2012 \& 2013 seasons. All treatments increased fruit set, the number of fruits / tree and yield in $\mathrm{kg} /$ tree, while decreased June drop and pre harvest drop compared to untreated trees (control).

\subsubsection{Fruit set percentage}

The treatment of $4 \%$ potassium chloride + $2 \%$ urea was the most effective treatment followed by the treatment of $2 \%$ potassium chloride $+2 \%$ urea in first season and $4 \%$ potassium chloride in second season. Untreated trees gave the least effects on fruit set.

\subsubsection{June drop percentage}

2 or $4 \%$ potassium chloride supplemented with $2 \%$ urea surpassed other treatments in reducing June drop percentage, while untreated trees exhibited the highest values of June drop. Other treatments came in-between.

\subsubsection{Pre-harvest drop}

Data indicated that in the first season, the treatment of $2 \%$ calcium chloride associated with $2 \%$ urea was the most effective treatment in reducing pre-harvest drop, while in the second season, the treatment of $2 \%$ potassium chloride $+2 \%$ urea exhibited the highest effect as compared to other treatments.

\subsubsection{Number of fruits per tree}

It is obvious from Table (1) that in both seasons of study, the treatment of $2 \%$ potassium chloride $+2 \%$ urea enhanced the number of fruits per tree followed by the treatment of $4 \%$ potassium chloride $+2 \%$ urea, while untreated trees showed the least values. Other treatments came in-between.

\subsubsection{Yield as kilogram per tree.}

Data showed that 2 or $4 \%$ potassium chloride supplemented with $2 \%$ urea increased yield significantly as kilogram per tree, while untreated trees showed the least values in both seasons.

The obtained results of tree fruiting are in line with the findings of El-Sharkawy, (2003) on olive where urea foliar spraying increased fruit set and yield, while decreased fruit drop. Desouky et al. (2009) found that spraying olive trees with calcium chloride increased fruit set. In addition, Antonio et al. (2011) found that pineapple fruit yield increased by potassium fertilization.

Table (1): Effect of some foliar application nutrient sprays on tree fruiting of Kalamata olive cultivar during 2012 \& 2013 seasons

\begin{tabular}{|c|c|c|c|c|c|c|c|c|c|c|}
\hline \multirow[t]{2}{*}{ Treatments } & \multicolumn{2}{|c|}{ Fruit set (\%) } & \multicolumn{2}{|c|}{ June drop (\%) } & \multicolumn{2}{|c|}{$\begin{array}{c}\text { Preharvest drop } \\
(\%)\end{array}$} & \multicolumn{2}{|c|}{ No. of fruits/tree } & \multicolumn{2}{|c|}{ Yield $(\mathrm{kg}) /$ tree } \\
\hline & 2012 & 2013 & 2012 & 2013 & 2012 & 2013 & 2012 & 2013 & 2012 & 2013 \\
\hline Control. & $\begin{array}{c}32.22 \\
\text { cd }\end{array}$ & $\begin{array}{c}38.86 \\
d\end{array}$ & $\begin{array}{c}46.08 \\
a\end{array}$ & $\begin{array}{c}45.35 \\
\mathrm{a}\end{array}$ & $\begin{array}{c}3.94 \\
\mathbf{a}\end{array}$ & $\begin{array}{c}2.31 \\
\mathbf{a b}\end{array}$ & $\begin{array}{l}3044.0 \\
\quad f\end{array}$ & $\begin{array}{c}3553.0 \\
\text { h }\end{array}$ & $\begin{array}{c}17.00 \\
\text { b }\end{array}$ & $\begin{array}{c}19.33 \\
\mathrm{~d}\end{array}$ \\
\hline $\begin{array}{l}1 \% \text { Calcium chloride } \\
21 \% \text {. }\end{array}$ & $\begin{array}{c}42.97 \\
\text { b }\end{array}$ & $\begin{array}{c}42.30 \\
\text { cd }\end{array}$ & $\begin{array}{c}31.85 \\
\text { b }\end{array}$ & $\begin{array}{c}27.90 \\
\text { b }\end{array}$ & $\begin{array}{c}1.85 \\
b\end{array}$ & $\begin{array}{c}2.86 \\
\mathrm{a}\end{array}$ & $\begin{array}{c}3543.0 \\
\text { cd }\end{array}$ & $\begin{array}{c}4422.0 \\
\mathrm{~g}\end{array}$ & $\begin{array}{c}19.67 \\
\text { b }\end{array}$ & $\begin{array}{c}22.00 \\
\text { cd }\end{array}$ \\
\hline $\begin{array}{l}2 \% \text { Calcium chloride } \\
21 \% \text {. }\end{array}$ & $\begin{array}{c}34.05 \\
\text { cd }\end{array}$ & $\begin{array}{c}42.29 \\
\text { cd }\end{array}$ & $\begin{array}{c}29.14 \\
b\end{array}$ & $\begin{array}{c}28.26 \\
b\end{array}$ & $\begin{array}{c}0.74 \\
\text { bc }\end{array}$ & $\begin{array}{c}2.22 \\
\text { ab }\end{array}$ & $\begin{array}{l}2948.0 \\
\text { f }\end{array}$ & $\begin{array}{c}5257.0 \\
\text { e }\end{array}$ & $\begin{array}{c}17.33 \\
\text { b }\end{array}$ & $\begin{array}{c}27.67 \\
\text { ab }\end{array}$ \\
\hline $2 \%$ Urea. & $\begin{array}{c}24.28 \\
\mathrm{e}\end{array}$ & $\begin{array}{c}43.20 \\
b-d\end{array}$ & $\begin{array}{c}22.20 \\
\text { c }\end{array}$ & $\begin{array}{c}23.38 \\
\text { b }\end{array}$ & $\begin{array}{c}\mathbf{0 . 8 1} \\
\text { bc }\end{array}$ & $\begin{array}{c}1.49 \\
\text { ab }\end{array}$ & $\begin{array}{l}3595.0 \\
\text { c }\end{array}$ & $\begin{array}{c}5265.0 \\
\mathrm{e}\end{array}$ & $\begin{array}{c}19.67 \\
b\end{array}$ & $\begin{array}{c}27.67 \\
\text { ab }\end{array}$ \\
\hline 2\% Potassium chloride. & $\begin{array}{c}36.23 \\
\text { c }\end{array}$ & $\begin{array}{c}41.55 \\
\text { cd }\end{array}$ & $\begin{array}{c}22.28 \\
\text { c }\end{array}$ & $\begin{array}{c}12.72 \\
\text { c }\end{array}$ & $\begin{array}{c}1.23 \\
\text { bc }\end{array}$ & $\begin{array}{c}\mathbf{0 . 5 3} \\
\text { cd }\end{array}$ & $\begin{array}{c}3369.0 \\
\mathrm{e}\end{array}$ & $\begin{array}{c}5426.0 \\
\text { d }\end{array}$ & $\begin{array}{c}17.33 \\
\text { b }\end{array}$ & $\begin{array}{c}29.00 \\
\text { ab }\end{array}$ \\
\hline 4\% Potassium chloride & $\begin{array}{c}37.08 \\
\text { c }\end{array}$ & $\begin{array}{l}49.30 \\
\text { ab }\end{array}$ & $\begin{array}{c}11.43 \\
d\end{array}$ & $\begin{array}{c}10.31 \\
\text { cd }\end{array}$ & $\begin{array}{c}0.50 \\
\text { bc }\end{array}$ & $\begin{array}{c}0.79 \\
\text { cd }\end{array}$ & $\begin{array}{c}4695.0 \\
b\end{array}$ & $\begin{array}{c}5601.0 \\
\text { c }\end{array}$ & $\begin{array}{c}25.33 \\
\text { a }\end{array}$ & $\begin{array}{c}30.00 \\
\text { ab }\end{array}$ \\
\hline $\begin{array}{l}1 \% \text { Calcium chloride } 21 \% \\
+2 \% \text { urea }\end{array}$ & $\begin{array}{c}28.46 \\
\text { de }\end{array}$ & $\begin{array}{c}46.03 \\
\text { a-c }\end{array}$ & $\begin{array}{c}12.92 \\
\text { d }\end{array}$ & $\begin{array}{c}10.22 \\
\text { cd }\end{array}$ & $\begin{array}{c}1.55 \\
\text { bc }\end{array}$ & $\begin{array}{c}0.34 \\
\text { cd }\end{array}$ & $\begin{array}{c}3449.0 \\
\text { de }\end{array}$ & $\begin{array}{l}5579.0 \\
\text { c }\end{array}$ & $\begin{array}{c}19.67 \\
b\end{array}$ & $\begin{array}{c}30.33 \\
\text { ab }\end{array}$ \\
\hline $\begin{array}{l}2 \% \text { Calcium chloride } \\
21 \%+2 \% \text { urea }\end{array}$ & $\begin{array}{c}32.08 \\
\text { cd }\end{array}$ & $\begin{array}{l}48.97 \\
\text { ab }\end{array}$ & $\begin{array}{c}19.69 \\
c\end{array}$ & $\begin{array}{c}6.17 \\
d\end{array}$ & $\begin{array}{c}0.00 \\
c\end{array}$ & $\begin{array}{c}0.79 \\
\text { cd }\end{array}$ & $\begin{array}{c}3419.0 \\
\text { de }\end{array}$ & $\begin{array}{c}5009.0 \\
\text { f }\end{array}$ & $\begin{array}{c}18.33 \\
b\end{array}$ & $\begin{array}{c}26.00 \\
\text { bc }\end{array}$ \\
\hline $\begin{array}{l}2 \% \text { Potassium chloride }+ \\
2 \% \text { urea }\end{array}$ & $\begin{array}{c}43.37 \\
\text { b }\end{array}$ & $\begin{array}{c}48.61 \\
\text { ab }\end{array}$ & $\begin{array}{c}10.21 \\
d\end{array}$ & $\begin{array}{c}5.67 \\
d\end{array}$ & $\begin{array}{c}0.41 \\
\text { bc }\end{array}$ & $\begin{array}{c}\mathbf{0 . 0 0} \\
\mathrm{d}\end{array}$ & $\begin{array}{c}5069.0 \\
\text { a }\end{array}$ & $\begin{array}{c}6102.0 \\
a\end{array}$ & $\begin{array}{c}26.33 \\
\mathbf{a}\end{array}$ & $\begin{array}{c}31.33 \\
\mathbf{a}\end{array}$ \\
\hline $\begin{array}{l}4 \% \text { Potassium chloride }+ \\
2 \% \text { urea }\end{array}$ & $\begin{array}{c}50.01 \\
\mathbf{a}\end{array}$ & $\begin{array}{c}\mathbf{5 0 . 2 3} \\
\mathbf{a} \\
\end{array}$ & $\begin{array}{c}9.01 \\
\text { d }\end{array}$ & $\begin{array}{c}5.02 \\
\text { d }\end{array}$ & $\begin{array}{c}\text { 0.77 } \\
\text { bc }\end{array}$ & $\begin{array}{c}2.15 \\
\mathbf{a b}\end{array}$ & $\begin{array}{c}758.0 \\
\text { b } \\
\end{array}$ & $\begin{array}{c}5974.0 \\
\text { b } \\
\end{array}$ & $\begin{array}{c}25.00 \\
\text { a } \\
\end{array}$ & $\begin{array}{c}32.33 \\
\mathrm{a} \\
\end{array}$ \\
\hline
\end{tabular}

Means followed by the same letter (s) are not significantly different at $5 \%$ level 


\subsection{Fruit physical characteristics}

Table (2) presents the effect of the treatments i.e., calcium chloride; potassium chloride and urea alone or in combination on fruit physical characteristics i.e., fruit weight, fruit length, fruit diameter, fruit shape and flesh/pit ratio during 2012 \& 2013 seasons.

\subsubsection{Fruit weight}

It is obvious that fruit weight was affected significantly by the treatments. The treatment of $2 \%$ calcium chloride was the superior, while $2 \%$ potassium chloride showed the lowest value in the first season. In the second season, untreated trees showed the highest value, while, $1 \%$ calcium chloride gave the least value. Other treatments came in-between.

\subsubsection{Fruit length}

The data indicated that in the first season, the treatment of $1 \%$ calcium chloride was the most effective; followed by $2 \%$ calcium chloride whereas, $2 \%$ potassium chloride $+2 \%$ urea gave the lowest value. While, $4 \%$ potassium chloride, followed by $2 \%$ potassium chloride followed by $1 \%$ calcium chloride $+2 \%$ urea were the most effective treatments in comparison with other treatments at the second season.

\subsubsection{Fruit diameter}

Table (2) showed that $2 \%$ calcium chloride gave the highest value of fruit diameter, while, the other treatments came in the second order without significant differences between them in the first season. In the second season, the treatment of $2 \%$ potassium chloride was superior to other treatments, followed by the treatment of $4 \%$ potassium chloride whereas the control (untreated trees) and other treatments came at the last with slight differences between them.

\subsubsection{Fruit shape}

In both seasons of the study, fruit shape was not affected by the tested treatments.

\subsubsection{Flesh/pit ratio}

Data showed that in both seasons, all tested treatments significantly affected flesh/pit ratio. The treatments of 2 or $4 \%$ potassium chloride, each associated with $2 \%$ urea were the most effective treatments, followed by $2 \%$ potassium alone in the first season. In the second season, $2 \%$ urea and $1 \%$ calcium chloride $+2 \%$ urea were the most effective treatments, while the lowest value came from $2 \%$ potassium chloride $+2 \%$ urea treatment. Other treatments came in-between.

The results of fruit physical characteristics are in harmony with the results of Fallahi et al. (2002) where foliar-application of urea at the highest rate had the heaviest fruits of "Fuji" apple trees. Besides, Porro et al. (2002) reported that foliar nutritional sprays can be a useful management practice to enhance apple fruit quality. In addition, Ben Mimoun et al. (2004) found that foliar fertilization of potassium in olive trees increased fruit weight and pit ratio.

Table (2): Effect of some foliar application nutrient sprays on fruit physical characteristics of Kalamata olive cultivar during 2012 \& 2013 seasons

\begin{tabular}{|c|c|c|c|c|c|c|c|c|c|c|}
\hline \multirow{2}{*}{ Treatments } & \multicolumn{2}{|c|}{$\begin{array}{c}\text { Fruit weight } \\
\text { (g.) }\end{array}$} & \multicolumn{2}{|c|}{$\begin{array}{c}\text { Fruit length } \\
(\mathrm{cm} .)\end{array}$} & \multicolumn{2}{|c|}{$\begin{array}{c}\text { Fruit diameter } \\
(\mathbf{c m} .)\end{array}$} & \multicolumn{2}{|c|}{$\begin{array}{c}\text { Fruit shape } \\
\text { (L/D) }\end{array}$} & \multicolumn{2}{|c|}{ Flesh/pit ratio } \\
\hline & 2012 & 2013 & 2012 & 2013 & 2012 & 2013 & 2012 & 2013 & 2012 & 2013 \\
\hline Control. & $\begin{array}{l}5.63 \\
\text { a-c }\end{array}$ & $\begin{array}{c}5.48 \\
\mathrm{a}\end{array}$ & $\begin{array}{l}3.04 \\
\text { a-c }\end{array}$ & $\begin{array}{c}2.87 \\
\text { cd }\end{array}$ & $\begin{array}{c}1.84 \\
\text { ab }\end{array}$ & $\begin{array}{c}1.85 \\
\text { cd }\end{array}$ & $\begin{array}{c}1.64 \\
\text { a }\end{array}$ & $\begin{array}{c}1.55 \\
\mathrm{a}\end{array}$ & $\begin{array}{c}5.67 \\
\text { cd }\end{array}$ & $\begin{array}{c}6.46 \\
b\end{array}$ \\
\hline $1 \%$ Calcium chloride $21 \%$.. & $\begin{array}{c}5.62 \\
\text { a-c }\end{array}$ & $\begin{array}{c}4.97 \\
\text { c }\end{array}$ & $\begin{array}{c}3.09 \\
\text { a }\end{array}$ & $\begin{array}{c}2.84 \\
d\end{array}$ & $\begin{array}{c}1.84 \\
\text { ab }\end{array}$ & $\begin{array}{c}1.84 \\
\text { d }\end{array}$ & $\begin{array}{c}1.68 \\
a\end{array}$ & $\begin{array}{c}1.55 \\
\mathbf{a}\end{array}$ & $\begin{array}{c}\text { 6.00 } \\
\text { ab }\end{array}$ & $\begin{array}{c}6.00 \\
d\end{array}$ \\
\hline $\begin{array}{l}2 \% \\
21 \% \text { Calcium }\end{array}$ & $\begin{array}{c}5.89 \\
\text { a }\end{array}$ & $\begin{array}{c}5.28 \\
\mathbf{a b}\end{array}$ & $\begin{array}{c}3.07 \\
\text { ab }\end{array}$ & $\begin{array}{l}2.91 \\
b-d\end{array}$ & $\begin{array}{c}1.86 \\
\text { a }\end{array}$ & $\begin{array}{c}1.87 \\
\text { cd }\end{array}$ & $\begin{array}{c}1.65 \\
\mathrm{a}\end{array}$ & $\begin{array}{c}1.55 \\
\mathrm{a}\end{array}$ & $\begin{array}{c}5.84 \\
\text { bc }\end{array}$ & $\begin{array}{c}6.13 \\
\text { cd }\end{array}$ \\
\hline $2 \%$ Urea. & $\begin{array}{l}5.52 \\
b-d\end{array}$ & $\begin{array}{l}5.27 \\
\text { a-c }\end{array}$ & $\begin{array}{l}3.02 \\
\text { b-d }\end{array}$ & $\begin{array}{c}2.86 \\
\text { cd }\end{array}$ & $\begin{array}{c}1.82 \\
\text { ab }\end{array}$ & $\begin{array}{c}1.85 \\
\text { cd }\end{array}$ & $\begin{array}{c}1.66 \\
\text { a }\end{array}$ & $\begin{array}{c}1.54 \\
\mathrm{a}\end{array}$ & $\begin{array}{c}5.54 \\
d\end{array}$ & $\begin{array}{c}6.90 \\
\mathbf{a}\end{array}$ \\
\hline $2 \%$ Potassium chloride. & $\begin{array}{c}5.15 \\
\mathrm{e}\end{array}$ & $\begin{array}{c}5.34 \\
\text { ab }\end{array}$ & $\begin{array}{c}3.00 \\
\text { cd }\end{array}$ & $\begin{array}{c}2.99 \\
\text { ab }\end{array}$ & $\begin{array}{c}1.81 \\
\text { ab }\end{array}$ & $\begin{array}{c}1.97 \\
\mathbf{a}\end{array}$ & $\begin{array}{c}1.66 \\
\mathrm{a}\end{array}$ & $\begin{array}{c}1.52 \\
\mathrm{a}\end{array}$ & $\begin{array}{l}\mathbf{6 . 0 3} \\
\text { ab }\end{array}$ & $\begin{array}{c}6.39 \\
\text { b }\end{array}$ \\
\hline $4 \%$ Potassium chloride. & $\begin{array}{l}5.43 \\
\text { b-e }\end{array}$ & $\begin{array}{c}5.35 \\
\text { ab }\end{array}$ & $\begin{array}{l}3.05 \\
\text { a-c }\end{array}$ & $\begin{array}{c}3.04 \\
\mathbf{a}\end{array}$ & $\begin{array}{c}1.83 \\
\text { ab }\end{array}$ & $\begin{array}{c}1.94 \\
\text { ab }\end{array}$ & $\begin{array}{c}1.66 \\
\text { a }\end{array}$ & $\begin{array}{c}1.56 \\
\mathrm{a}\end{array}$ & $\begin{array}{c}6.02 \\
\text { ab }\end{array}$ & $\begin{array}{c}6.15 \\
\text { cd }\end{array}$ \\
\hline $\begin{array}{l}1 \% \text { Calcium chloride } 21 \% \\
+2 \% \text { urea }\end{array}$ & $\begin{array}{c}5.71 \\
\text { ab }\end{array}$ & $\begin{array}{c}5.46 \\
\text { ab }\end{array}$ & $\begin{array}{l}3.04 \\
\text { a-c }\end{array}$ & $\begin{array}{c}2.98 \\
\text { ab }\end{array}$ & $\begin{array}{c}1.83 \\
\text { ab }\end{array}$ & $\begin{array}{c}1.91 \\
\text { a-c }\end{array}$ & $\begin{array}{c}1.67 \\
\mathbf{a}\end{array}$ & $\begin{array}{c}1.56 \\
\mathrm{a}\end{array}$ & $\begin{array}{c}5.98 \\
\text { ab }\end{array}$ & $\begin{array}{c}6.80 \\
\mathbf{a}\end{array}$ \\
\hline $\begin{array}{l}2 \% \text { Calcium chloride } 21 \% \\
+2 \% \text { urea } \\
\end{array}$ & $\begin{array}{l}5.37 \\
\text { b-e }\end{array}$ & $\begin{array}{l}5.18 \\
\text { a-c }\end{array}$ & $\begin{array}{c}2.97 \\
\text { de }\end{array}$ & $\begin{array}{c}2.87 \\
\text { cd }\end{array}$ & $\begin{array}{c}1.81 \\
\text { ab }\end{array}$ & $\begin{array}{c}1.87 \\
\text { cd }\end{array}$ & $\begin{array}{c}1.64 \\
\mathrm{a}\end{array}$ & $\begin{array}{c}1.53 \\
\mathrm{a}\end{array}$ & $\begin{array}{c}5.71 \\
\text { cd }\end{array}$ & $\begin{array}{c}6.33 b \\
\mathrm{c}\end{array}$ \\
\hline $\begin{array}{l}2 \% \text { Potassium chloride + } \\
2 \% \text { urea }\end{array}$ & $\begin{array}{c}5.19 \\
\text { de }\end{array}$ & $\begin{array}{l}5.15 \\
\text { bc }\end{array}$ & $\begin{array}{c}2.93 \\
\mathrm{e}\end{array}$ & $\begin{array}{l}2.90 \\
\text { b-d }\end{array}$ & $\begin{array}{c}1.78 \\
b\end{array}$ & $\begin{array}{l}1.90 \\
\text { a-d }\end{array}$ & $\begin{array}{c}1.65 \\
\mathbf{a}\end{array}$ & $\begin{array}{c}1.52 \\
\mathrm{a}\end{array}$ & $\begin{array}{c}6.08 \\
\mathbf{a}\end{array}$ & $\begin{array}{c}5.95 \\
\mathrm{~d}\end{array}$ \\
\hline $\begin{array}{l}4 \% \text { Potassium chloride }+ \\
2 \% \text { urea }\end{array}$ & $\begin{array}{l}5.27 \\
\text { c-e }\end{array}$ & $\begin{array}{c}5.41 \\
\text { ab }\end{array}$ & $\begin{array}{l}3.02 \\
\text { b-d }\end{array}$ & $\begin{array}{c}2.96 \\
\text { a-c }\end{array}$ & $\begin{array}{c}1.81 \\
\mathbf{a b}\end{array}$ & $\begin{array}{l}1.90 \\
b-d\end{array}$ & $\begin{array}{c}1.67 \\
\mathrm{a}\end{array}$ & $\begin{array}{c}1.55 \\
\mathrm{a}\end{array}$ & $\begin{array}{c}6.08 \\
\mathbf{a}\end{array}$ & $\begin{array}{c}6.25 \\
\text { bc }\end{array}$ \\
\hline
\end{tabular}

Means followed by the same letter (s) are not significantly different at $5 \%$ level. 


\subsection{Fruit oil percentage}

Fruit oil content as fruit fresh or dry weight is tabulated in Table (3). In both seasons, all treatments increased fruit oil percentage as fruit fresh weight or fruit dry weight compared with the control (untreated trees). containing: nirogen and potassium(Olive-NutriVant ) increased fruit oil content. El-Sharkawy (2003) found that spraying olive trees with urea increased oil percentage in olive fruits; Desouky et al. (2009) found that spraying three cultivars of olive with calcium chloride increased fruit oil

Table (3): Effect of some foliar application nutrient sprays on oil percentage of Kalamata olive cultivar during $2012 \& 2013$ seasons.

\begin{tabular}{|l|c|c|c|c|}
\hline \multirow{2}{*}{ Treatments } & \multicolumn{2}{c|}{ Oil percentage / fresh weight } & \multicolumn{2}{c|}{ Oil percentage / dry weight } \\
\cline { 2 - 5 } & 2012 & 2013 & 2012 & 2013 \\
\hline Control. & $16.84 \mathrm{e}$ & $16.46 \mathrm{e}$ & $46.39 \mathrm{~cd}$ & $46.29 \mathrm{~d}$ \\
\hline $1 \%$ Calcium chloride 21\%. & $16.92 \mathrm{de}$ & $16.57 \mathrm{de}$ & $48.72 \mathrm{ab}$ & $46.94 \mathrm{~cd}$ \\
\hline $2 \%$ Calcium chloride 21\%. & $17.22 \mathrm{c}$ & $16.66 \mathrm{c}-\mathrm{e}$ & $45.69 \mathrm{~d}$ & $46.69 \mathrm{~d}$ \\
\hline $2 \%$ Urea. & $17.14 \mathrm{~cd}$ & $16.57 \mathrm{de}$ & $46.96 \mathrm{~b}-\mathrm{d}$ & $47.56 \mathrm{~b}-\mathrm{d}$ \\
\hline $2 \%$ Potassium chloride. & $17.68 \mathrm{a}$ & $16.81 \mathrm{~cd}$ & $49.63 \mathrm{a}$ & 48.88 a-c \\
\hline $4 \%$ Potassium chloride & $17.17 \mathrm{~cd}$ & $17.86 \mathrm{a}$ & $47.54 \mathrm{a}-\mathrm{d}$ & $49.92 \mathrm{a}$ \\
\hline $1 \%$ Calcium chloride $21 \%+2 \%$ urea & $17.36 \mathrm{bc}$ & $16.96 \mathrm{c}$ & $49.75 \mathrm{a}$ & $46.87 \mathrm{~cd}$ \\
\hline $2 \%$ Calcium chloride $21 \%+2 \%$ urea & $17.09 \mathrm{c}-\mathrm{e}$ & $17.48 \mathrm{~b}$ & $46.69 \mathrm{~b}-\mathrm{d}$ & $49.55 \mathrm{ab}$ \\
\hline $2 \%$ Potassium chloride $+2 \%$ urea & $17.36 \mathrm{bc}$ & $17.74 \mathrm{ab}$ & $48.23 \mathrm{a}-\mathrm{c}$ & $49.35 \mathrm{ab}$ \\
\hline $4 \%$ Potassium chloride $+2 \%$ urea & $17.53 \mathrm{ab}$ & $17.65 \mathrm{ab}$ & $47.53 \mathrm{a}-\mathrm{d}$ & $49.89 \mathrm{a}$ \\
\hline
\end{tabular}

Means followed by the same letter (s) are not significantly different at $5 \%$ level

\subsubsection{Fruit oil percentage/ fresh weight}

In the first season, the treatment of $2 \%$ potassium chloride induced the highest values, whereas $4 \%$ potassium chloride $+2 \%$ urea came at the second order, followed by $1 \%$ calcium chloride $+2 \%$ urea and $2 \%$ potassium chloride + $2 \%$ urea without significant differences between them. In the second season, $4 \%$ potassium chloride was the superior treatment, whereas, both $2 \%$ potassium chloride and $4 \%$ potassium chloride each associated with $2 \%$ urea occupied the second order without significant differences between them.

\subsubsection{Fruit oil percentage/ dry weight}

In the first season, the treatment of $1 \%$ calcium chloride associated with $2 \%$ urea induced the highest values of oil percentage followed by $2 \%$ potassium chloride alone without significant differences between them, followed by $1 \%$ calcium chloride. Other treatments induced similar results. Meanwhile, in the second season, both the treatments of $4 \%$ potassium chloride alone and 4 $\%$ potassium chloride $+2 \%$ urea induced the highest values without significant differences between them, followed by both $2 \%$ calcium chloride and $2 \%$ potassium chloride each supplemented with $2 \%$ urea without significant differences between them.

The results of fruit oil content came in line with those of Wiesman et al. (2002) who found that spraying olive trees with a compound content and improved oil quality and Hegazi et al. (2011) found that spraying olive trees with nitrogen or potassium increased fruit oil content.

\subsubsection{Leaves NPK content}

Percentages of N, P and K of Kalamata olive cv. leaves are reported in Table (4) for both seasons. Regarding leaves nitrogen content, it was revealed that in the first season, the treatment of $2 \%$ calcium chloride induced the highest value, followed by the control without significant differences between them, followed by $2 \%$ calcium chloride $+2 \%$ urea, while $2 \%$ potassium chloride $+2 \%$ urea recorded the lowest value. Other treatments came in between. In the second season, $2 \%$ potassium chloride associated with $2 \%$ urea was superior to other treatments, followed by the treatment of $4 \%$ potassium chloride supplemented with $2 \%$ urea and $2 \%$ calcium chloride associated with $2 \%$ urea, while, the other treatments came in-between.

As regard to phosphorus content in the first season, it was found that the treatment of $4 \%$ potassium chloride was superior to the other treatments, while in the second season, $2 \%$ potassium chloride induced the highest values of phosphorus percentages in comparison with the other treatments.

Concerning leaf potassium content, it is obvious from Table (4) that in the first season, the untreated trees (control) gave the highest value, 


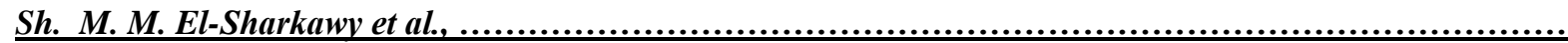

Table (4): Effect of some foliar application nutrient sprays on leaves NPK of Kalamata olive cultivar during $2012 \& 2013$ seasons.

\begin{tabular}{|c|c|c|c|c|c|c|}
\hline \multirow{2}{*}{ Treatments } & \multicolumn{2}{|c|}{$\mathbf{N}(\%)$} & \multicolumn{2}{|c|}{$\mathbf{P}(\%)$} & \multicolumn{2}{|c|}{$\mathrm{K}(\%)$} \\
\hline & 2012 & 2013 & 2012 & 2013 & 2012 & 2013 \\
\hline Control. & $\begin{array}{c}2.003 \\
\mathrm{a}\end{array}$ & $\begin{array}{c}1.433 \\
\text { cd }\end{array}$ & $\begin{array}{c}0.253 \\
\text { b }\end{array}$ & $\begin{array}{c}0.257 \\
\text { b }\end{array}$ & $\begin{array}{c}1.183 \\
a\end{array}$ & $\begin{array}{c}0.823 \\
\text { ab }\end{array}$ \\
\hline $1 \%$ Calcium chloride $21 \%$. & $\begin{array}{c}1.577 \\
\text { cd }\end{array}$ & $\begin{array}{c}1.500 \\
\text { c }\end{array}$ & $\begin{array}{c}0.180 \\
\text { c }\end{array}$ & $\begin{array}{c}0.100 \\
\text { e }\end{array}$ & $\begin{array}{c}0.967 \\
\text { e }\end{array}$ & $\begin{array}{c}0.920 \\
\mathrm{a}\end{array}$ \\
\hline $2 \%$ Calcium chloride $21 \%$. & $\begin{array}{c}2.053 \\
\text { a }\end{array}$ & $\begin{array}{c}1.167 \\
\text { e }\end{array}$ & $\begin{array}{c}\mathbf{0 . 1 3 0} \\
\text { e }\end{array}$ & $\begin{array}{c}0.09 \\
\text { e }\end{array}$ & $\begin{array}{c}1.170 \\
\text { ab }\end{array}$ & $\begin{array}{c}0.797 \\
\text { a-c }\end{array}$ \\
\hline $2 \%$ Urea. & $\begin{array}{c}1.620 \\
\text { b-d }\end{array}$ & $\begin{array}{c}1.667 \\
\text { b }\end{array}$ & $\begin{array}{c}\mathbf{0 . 1 3 0} \\
\text { e }\end{array}$ & $\begin{array}{c}0.100 \\
\text { e }\end{array}$ & $\begin{array}{c}0.970 \\
\text { e }\end{array}$ & $\begin{array}{c}0.483 \\
d\end{array}$ \\
\hline 2\% Potassium chloride. & $\begin{array}{c}1.520 \\
\text { de }\end{array}$ & $\begin{array}{c}1.707 \\
\text { b }\end{array}$ & $\begin{array}{c}0.160 \\
d\end{array}$ & $\begin{array}{c}0.313 \\
\mathrm{a}\end{array}$ & $\begin{array}{c}1.107 \\
\text { cd }\end{array}$ & $\begin{array}{c}0.577 \\
\text { cd }\end{array}$ \\
\hline 4\% Potassium chloride & $\begin{array}{c}1.720 \\
\text { b-d }\end{array}$ & $\begin{array}{c}1.447 \\
\text { cd }\end{array}$ & $\begin{array}{c}0.417 \\
\text { a }\end{array}$ & $\begin{array}{c}0.160 \\
\text { d }\end{array}$ & $\begin{array}{c}1.070 \\
\text { d }\end{array}$ & $\begin{array}{c}0.727 \\
\text { a-c }\end{array}$ \\
\hline $1 \%$ Calcium chloride $21 \%+2 \%$ urea & $\begin{array}{c}1.370 \\
\text { e }\end{array}$ & $\begin{array}{c}1.387 \\
\text { d }\end{array}$ & $\begin{array}{c}0.167 \\
\text { cd }\end{array}$ & $\begin{array}{c}0.100 \\
\text { e }\end{array}$ & $\begin{array}{c}1.083 \\
d\end{array}$ & $\begin{array}{c}0.910 \\
\text { ab }\end{array}$ \\
\hline $2 \%$ Calcium chloride $21 \%+2 \%$ urea & $\begin{array}{c}1.800 \\
b\end{array}$ & $\begin{array}{c}1.673 \\
\text { b }\end{array}$ & $\begin{array}{c}\mathbf{0 . 1 3 7} \\
\text { e }\end{array}$ & $\begin{array}{c}\mathbf{0 . 1 0 0} \\
\mathrm{e}\end{array}$ & $\begin{array}{c}1.117 \\
\text { b-d }\end{array}$ & $\begin{array}{c}0.680 \\
\text { b-d }\end{array}$ \\
\hline $2 \%$ Potassium chloride $+2 \%$ urea & $\begin{array}{c}1.050 \\
\text { f }\end{array}$ & $\begin{array}{c}2.053 \\
\text { a }\end{array}$ & $\begin{array}{c}\mathbf{0 . 1 3 0} \\
\text { e }\end{array}$ & $\begin{array}{c}0.190 \\
\text { c }\end{array}$ & $\begin{array}{c}0.963 \\
\text { e }\end{array}$ & $\begin{array}{c}0.833 \\
\text { ab }\end{array}$ \\
\hline $4 \%$ Potassium chloride $+2 \%$ urea & $\begin{array}{c}1.750 \\
\text { bc }\end{array}$ & $\begin{array}{c}1.723 \\
b\end{array}$ & $\begin{array}{c}0.120 \\
\mathrm{e}\end{array}$ & $\begin{array}{c}0.150 \\
d\end{array}$ & $\begin{array}{c}1.163 \\
\text { a-c }\end{array}$ & $\begin{array}{c}0.737 \\
\text { a-c }\end{array}$ \\
\hline
\end{tabular}

Means followed by the same letter (s) are not significantly different at $5 \%$ level

followed by $2 \%$ calcium chloride. In the second season, $1 \%$ calcium chloride induced the highest value, followed by $1 \%$ calcium chloride $+2 \%$ urea. The least value resulted from $2 \%$ urea and the other treatments came in-between.

The results of leaf NPK are in line with the findings of Sánchez-Zamora and FernándezEscobar (2002) on olive, who found that spraying urea on olive trees compared to nitrogen soil application improved leaf $\mathrm{N}$ concentration. In addition,Weinbaum et al., (2002) reported that foliar-applied macronutrients, such as $\mathrm{N}$ and $\mathrm{K}$, may be anticipated when soil nutrient acquisition is inadequate to meet tree nutrient demand. Significant quantities of nutrients can be delivered foliarly, absorbed from leaf surfaces and translocated to sites of utilization within the tree.

\section{Recommendations}

It can be recommended that spraying olive trees cv. Kalamata with $1 \%$ or $2 \%$ calcium chloride; 2 $\%$ or $4 \%$ potassium chloride and $2 \%$ urea each alone or in combination increased fruiting; fruit oil content and improved fruit quality.

\section{REFERENCES}

Andrews P. K. (2002). How foliar - applied nutrients affect stresses in perennial fruit plants. Acta Hort. (ISHS) 594:49-55.

Antonio L., Quaggio L. A., Cantarella H. and Mellis E. V. (2011). Potassium fertilization for pineapple: effect on plant growth and fruit yield. Rev. Bras. Frutic. Jaboticabal - SP, V. 33, N. 2, p. 618-626, June 2011.

A.O.A.C. (1980). Official Methods Analysis $\left(12^{\text {th }}\right.$ Ed). Association Of Official Analytical Chemists, Washington DC.

Ben Mimoun M., Loumi O., Ghrab M., Latiri K. and Hellali R. (2004). Foliar potassium application on olive tree. Regional workshop on potassium and fertigation development in west Asia and North Africa; Rabat, Morocco, 24-28 November, 2004.

Chapman H.D. and Pratt P. F. (1978). "Methods of Analysis for Soils, Plants and Waters" Univ. California, Div. Agric. Sci. 17-150.

Desouky I. M., Haggag L. F., Abd El-Migeed M.M.M., Kishk Y.F.M. and El-Hady E.S. (2009). Effect of boron and calcium nutrient sprays on fruit set, oil content and oil quality of some olive oil cultivars. World J. Agric. Sci.: 2, 180-185.

Duncan D. B. (1955). Multiple range and multiple F. Test. Biometrics 11:1-42.

El-Sayed M. E. and Abou Shanab I. S. (2011). Olive Growing in Egypt. A pamphlet publication, Hort. Res. Inst., Giza, Egypt.

El-Sharkawy Sh. M.M. (2003). Effect of pruning levels and boron and urea sprays on fruit set yield and fruit quality of olive trees. Annals of Agric. Sci. Moshtohor, 41 
(3):1307-1322.

Fallahi E., Khemira H., Righetti T. I. and Azarenko A. N. (2002). Influence of foliar application of urea on tree growth, fruit quality, leaf minerals, and distribution of urea-drived nitrogen in apples. Acta Hort. 594:603-610.

Fernandez-Escobar R., Moreno R. and SanchezZamora M. A. (2004). Nitrogen dynamics in the olive bearing shoot. Hort.Sci. 39:1406-1411.

Freeman M. , Uriu K. and Hartman H. T. (2005). In: Diagnosing and correcting nutrient problems, Pp.83-92.

Haj Sulaiman A. (2009). Effect of foliar application with boron and calcium on fruit quality of two olive varieties "Sorani and Kaissi". General Commission for Scientific Agricultural Research, olive Research Department. Aleppo University, Faculty of Agriculture.

Hegazi E. S., Mohamed S. M., El-Sonbaty M.R., Abd El-Naby S. K. M. and El-Sharony T. F. (2011). Effect of potassium nitrate on vegetative growth, nutritional status, yield and fruit quality of olive cv. "Picual". J. Hort. Sci. \& Ornament. Plants 3 (3): 252-258.

Hussein A. H. A. (2008). Respnse of Manzanillo olive "Olea europaea, L." Cultivar to irrigation regime and potassium fertigation under Tabouk condition, Saudi Arabia. J. Agron., 7: 285-296.

Porro D., Dorigatti C. and Ramponi M. (2002).
Can foliar application modify nutritional status and improve fruit quality results on apple in northeastern Italy. Acta Hort. 594:521-526.

Sánchez-Zamora M. A. and Fernández-Escobar R. (2002). The effect of foliar vs. soil application of urea to olive trees. Acta Hort. 594:675-678

Snedecor G.W. and Cochran W.G. (1980). Statistical Methods. $7^{\text {th }}$ ed. Iowa State Univ. Press, Ames, Iowa, U.S.A PP. 507.

Tavares B., Domingos P., Dias P. N., Feijo J. A. and Bicho A. (2011). The essential role of anionic transport in plant cells: the pollen tube as a case study J. Exp. Bot. 62 (7): 2273-2298.

Therios I. (2006). Mineral nutrition of olive trees. Proc. $2^{\text {nd }}$ Intil. Seminar Recent Advances Olive Industry. Pp.403-410.

Weinbaum S. A., Brown P. H. and Johnson R.S. (2002). Application of selected macronutrients $(\mathrm{N}, \mathrm{K})$ in deciduous orchards: physiological and agro technical perspectives. Acta Hort.(ISHS) 594:59-64.

Wide S. A., Corey R. B., Lyer J. G. and Vioget G.K. (1985)."Soil and Plants Analysis for tree culture $3^{\text {rd }}$ ed". Oxford IBH publishing Co., New Delhi, Pp 93-116.

Wiesman Z., Ronen A., Ankarion Y., Novikov V., Maranz S., Chpagain B. and Abramovich Z. (2002). Effect of olivenutri-vant on yield and quality of olives and oil. Acta Hort. (ISHS) 594:557-562.

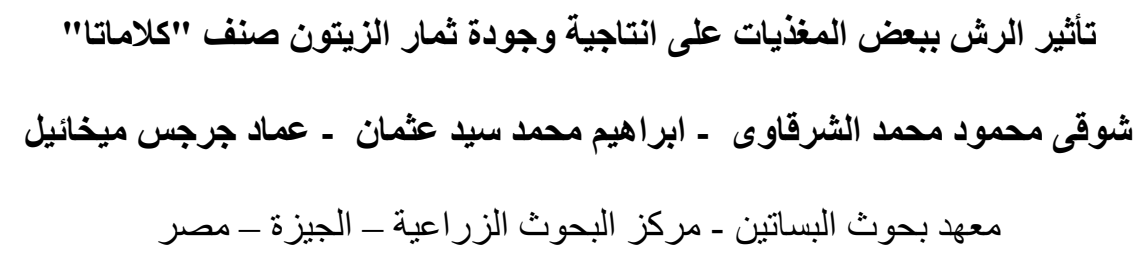

\footnotetext{
ملخص

أجريت هذه الدر اسة خلال موسمى 2012 و 2013 بمزرعة خاصة عند الكيلو 64 طريق مصر /إسكندرية الصحر اوى وذللك

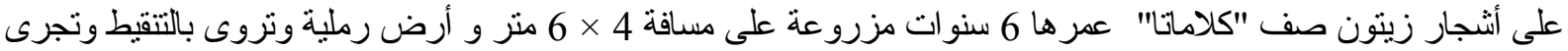

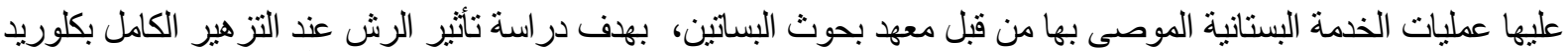

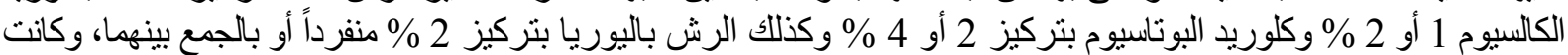

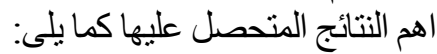

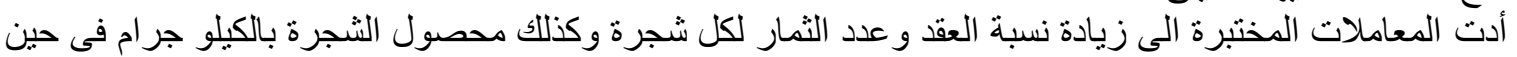

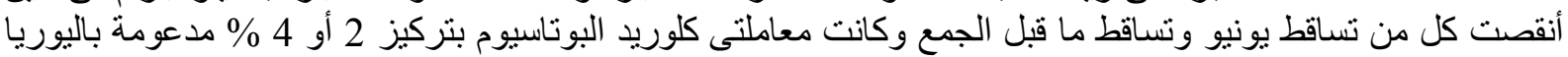

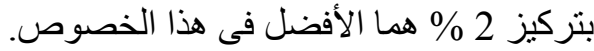

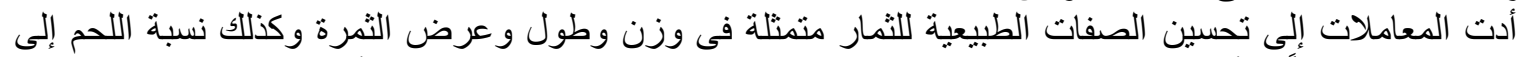

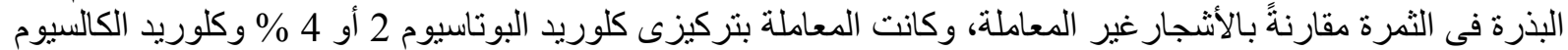
1 أو 2 \% منفردة أو مدعمة باليوريا بتركيز 2 \% هي الأنى الأفضل.
} 


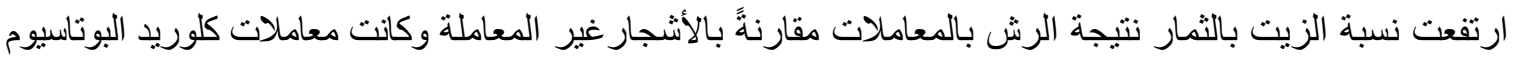

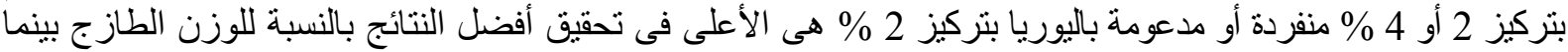

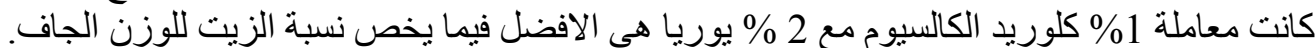

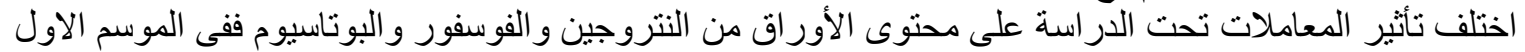

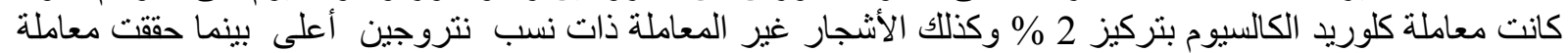

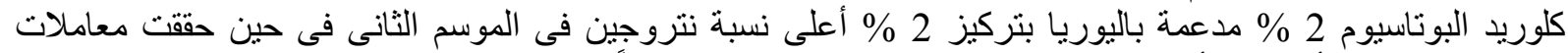

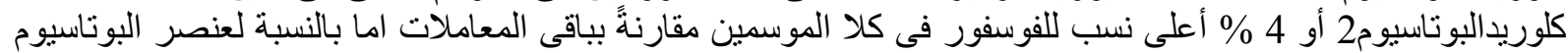

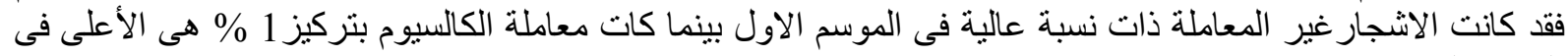

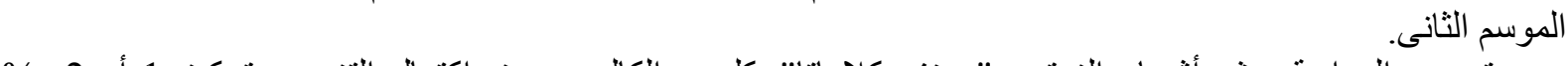

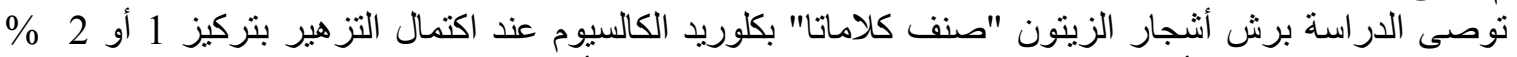
وكلوريد البوتاسيوم بتركيز 2 أو 4 \% و اليوريا بتركيز 2 \% كل على على حده أو فى تركيبات بينهما لزيادة انتاجية الاشجار

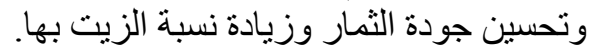
المجلة العلمية لكلية الزراعة - جامعة القاهرة ـ المجلد (65) العدد الثالث (يوليو 2014) :295-295. 